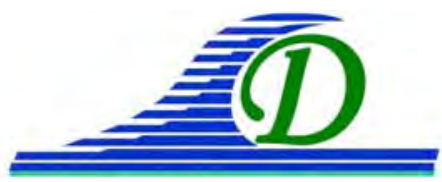

XIII ${ }^{\text {èmes }}$ Journées Nationales Génie Côtier - Génie Civil

Dunkerque, 2-4 juillet 2014

DOI:10.5150/jngcgc.2014.008 @ Editions Paralia CFL

disponible en ligne - $h$ ttp://www.paralia.fr - available online

\title{
Les nouveaux produits de courants de marée et services proposés par le SHOM
}

\author{
Stéphanie DESMARE ${ }^{1}$, Nicolas WEBER $^{2}$
}

1. Service hydrographique et océanographique de la marine, Département hydrodynamique côtière (HDC) - Courants, SHOM/DOPS/MIP/HDC, 13, rue du Chatellier, CS92803, 29228 Brest Cedex 2, France. stephanie.desmare@shom.fr

2. Service hydrographique et océanographique de la marine, Département hydrodynamique côtière (HDC) - Courants, SHOM/DOPS/MIP/HDC, 13, rue du Chatellier, CS92803, 29228 Brest Cedex 2, France.nicolas.weber@shom.fr

\section{Résumé :}

Dans le domaine maritime, la connaissance et la prévision des courants sont nécessaires pour répondre aux besoins d'informations nautiques et appréhender leurs impacts sur la sécurité de la navigation, sur les opérations (en surface et sous-marines) et sur les autres activités maritimes.

Depuis la diffusion des atlas de courants de marée du SHOM couvrant l'ensemble des côtes françaises de la Manche et de l'Atlantique, puis la parution du cédérom associé en 2005, les demandes d'informations de plus en plus précises sur cette thématique ont été croissantes.

L'élaboration de modèles numériques de courants de marée en 3D et l'évolution des besoins (défense et civils : énergies marines renouvelables, activités maritimes, pêche, aquaculture, etc.) conduisent à définir de nouveaux produits qui fournissent des informations pertinentes sur les courants de marée, en qualifiant les courants de surface, mais également de fond et à des immersions données le long de la colonne d'eau.

Cet article présente les derniers produits de courant de marée élaborés au SHOM : d'une part la nouvelle édition de l'atlas de courants pour le Pas-de-Calais (sortie prévue en 2014) et le produit de courant 3D Manche disponible depuis mars 2013. Une dernière partie présente le nouveau portail data.shom.fr et ses futures offres de service de courants de marée.

Mots-clés : Hydrodynamique côtière, Marée, Courants, Modélisations, Produits SIG.

\section{Introduction}

La mise à jour de l'atlas de courant de marée du Pas de Calais, 25 ans après sa première édition, concrétise un ensemble de travaux menés depuis plusieurs années au SHOM.

Premier d'une génération d'ouvrages de courant réalisés à partir d'un modèle numérique, l'atlas du Pas-de-Calais a permis au SHOM d'envisager les améliorations qui ont pu être apportées à la réalisation des huit autres atlas régionaux diffusés sous format papier. 


\section{Thème 1 - Hydrodynamique côtière}

Les outils techniques, ainsi que les méthodes développées pour garantir la qualité des informations disponibles au SHOM, sont abordées dans la première partie de cet article. L'évolution de ces outils et des méthodes de calcul ont permis par la suite d'envisager une extension de la gamme des produits de courants de marée avec les produits de courants de marée 3D (DESMARE, 2011). Ces derniers fournissent des informations plus détaillées à chaque point de maille et une meilleure description des processus côtiers. La mise à disposition des produits sous formats numériques permet dorénavent une exploitation plus aisée avec les logiciels utilisables dans le domaine des systèmes d'information géographique et des logiciels de navigation.

\section{Phases de réalisation des atlas de courants de marée}

L'intérêt principal des atlas régionaux est de présenter aux utilisateurs les informations de courants de marée de la façon la plus pratique possible. Chaque ouvrage couvre une zone définie avec un port de référence et fournit les informations de courants de marée (orientation et vitesse du courant à chaque heure de marée en vive-eau moyenne et morte eau moyenne).

Les diverses considérations sur les besoins ont conduit à réaliser les ouvrages en développant une méthode de mise en forme quasi automatique, l'accent étant mis sur une présentation qui puisse satisfaire la majorité des utilisateurs.

Les principales étapes de la procédure suivie pour réaliser les produits des courants de marées sont expliquées dans cette partie.

\subsection{Les données en entrée}

Les possibilités offertes par l'évolution des moyens de calcul et l'amélioration de la connaissance scientifique ont permis de développer un système de prévision des courants de marée basé sur la simulation numérique de la propagation de l'onde de marée dans un domaine maritime. La modélisation des courants en 2D et maintenant en 3D à l'aide de la chaîne de calcul Telemac-Mascaret (développé par le laboratoire d'hydraulique de Saint-Venant, http://www.opentelemac.org/2 permet d'obtenir des informations denses et une meilleure description des processus côtiers (NICOLLE \& PINEAU, 2009).

Les atlas du SHOM sont dès lors plus précis dans leur ensemble et plus cohérents que ceux générés uniquement à partir de mesures in situ, ponctuelles et locales.

Les mises à jour des atlas de courants de marée bénéficient des améliorations continues des modèles hydrodynamiques réalisés par le SHOM (NICOLLE, 2010a,b \& 2011a,b) permettant leur conception et des meilleures connaissances issues des multiples campagnes hydrographiques et à l'enrichissement continu des bases de données d'environnement. Ces évolutions se traduisent dans les produits de courants par une amélioration de la représentation. En effet, le modèle s' appuie sur un maillage triangulaire à maille variable permettant un raffinement dans les zones complexes 


\section{XIII ${ }^{\text {èmes }}$ Journées Nationales Génie Côtier - Génie Civil \\ Dunkerque, 2-4 juillet 2014}

(chenaux, zone portuaire, zones à topographie accidentée) et une résolution spatiale plus dense quelle que soit l' échelle (100 km au large, quelques dizaines de mètres à la côte, voir figure 1).

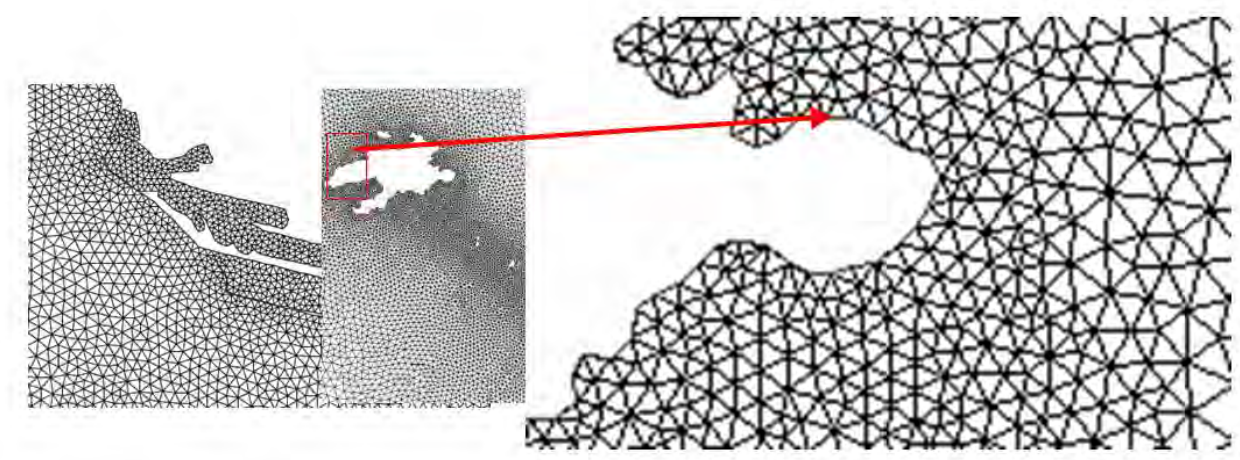

Figure 1. Exemples de maillages et de raffinements dans les zones présentant un intérêt particulier (notamment pour la navigation : ports, chenaux).

Tableau 1. Résolutions spatiales des différents modèles de courant de marée 3D mis en œuvre depuis 2009 au SHOM.

\begin{tabular}{lll}
\hline Modèle & Résolution verticale & Résolution horizontale \\
\hline Rade de Brest & 10 plans & $10 \mathrm{~m}$ à la côte à $500 \mathrm{~m}$ au large \\
Manche & 10 plans & $100 \mathrm{~m}$ à la côte à $20 \mathrm{~km}$ au large \\
Baie de Somme / Pas de Calais & 10 plans & $13 \mathrm{~m}$ à $6 \mathrm{~km}$ \\
Baie de Seine & 10 plans & $15 \mathrm{~m}$ à $7 \mathrm{~km}$ \\
Mer d'Iroise & 10 plans & $50 \mathrm{~m}$ à la côte à $10 \mathrm{~km}$ au large \\
Loire & 13 plans & $50 \mathrm{~m}$ à la côte à $7 \mathrm{~km}$ au large \\
\hline
\end{tabular}

\subsection{Les phases de réalisation des produits}

Les modèles validés par comparaison aux mesures in situ (hauteurs d'eau issues de marégraphes et vitesses de courant issus des courantomètres) sont à la base des produits de courants publiés par le SHOM. Ces ouvrages cartographient la direction et l'intensité des courants de marée à chaque heure par rapport à la pleine mer ou la basse mer d'un port de référence.

Ces produits de courants de marée répondent aux besoins de la navigation, de la plongée et de la pêche, ils constituent également des données d'entrée cruciales dans le cadre d'études environnementales : projets de rejets en mer, aménagements portuaires par exemple.

Plusieurs phases de traitements sont nécessaires à la réalisation d'un produit de courants de marée :

- Détermination des zones d'intérêts et choix de l'emprise de la planche du cadre général du produit et des éventuels zooms,

- Choix de la résolution spatiale en fonction des zones de zoom, 
- Choix du niveau vertical que l'on souhaite représenter (surface libre, mi-profondeur, fond),

- Calcul du courant maximal sur la grille d'origine en considérant chaque échéance de temps des sorties du modèle (5 à $10 \mathrm{mn}$ ) et cartographie du courant maximal sur la vue générale et les zooms,

- Identification des zones de forts courants maximaux et de fortes variations des courants,

- Choix des heures de pleine mer aux ports de référence,

- Génération des cartes de vecteurs de courant, choix d'une palette de couleurs,

- Contrôle visuel,

- Conception des fonds de carte,

- Transformation des fichiers de données en fichiers SIG,

- Production des cartes, génération de l'ouvrage en PDF (pour les atlas)

- Description et documentation.

\section{Résultats}

\subsection{Atlas de courant du Pas-de-Calais - Baie de Somme (557)}

Dans le Pas-de-Calais, les courants de marée ont un caractère alternatif marqué, de direction analogue en morte-eau et en vive-eau. Le choix d'une planche horaire unique pour la représentation des courants par coefficients de marée 45 et 95 a donc été gardé comme pour le précédent atlas.

Pour cette nouvelle édition du produit Pas-de-Calais, la couverture géographique a été étendue vers la Baie de Somme. Le produit couvre donc la zone allant de Dunkerque à Fécamp. Trois autres planches correspondent généralement à des zooms faits sur les parties des abords des ports de Dunkerque et de Calais (A), de Boulogne (B) et de Dieppe (C) (voir figure 2).
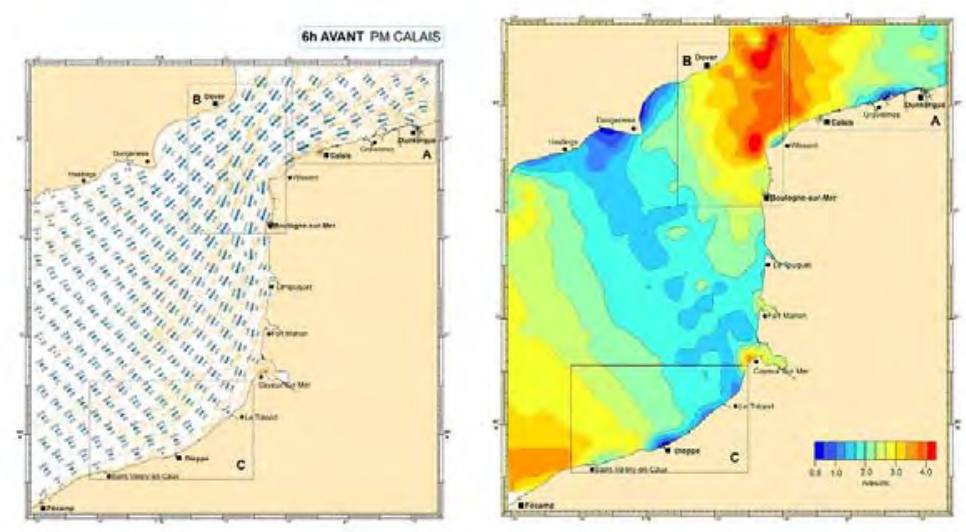

Figure 2. Emprise de la nouvelle édition de l'atlas de courant-Pas-de-Calais - Baie de Somme- 557. 


\section{XIII ${ }^{\text {èmes }}$ Journées Nationales Génie Côtier - Génie Civil \\ Dunkerque, 2-4 juillet 2014}

Cette nouvelle représentation apporte d'avantage d'informations à l'utilisateur qui, jusqu'ici ne disposait pour ces zooms que d'informations partielles aux points de mesures existants (voir figure 3).

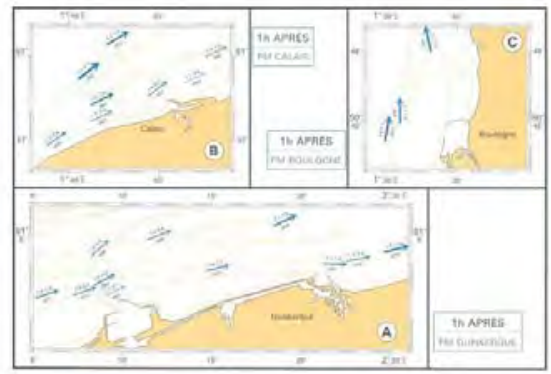

Atlas- édition 1988

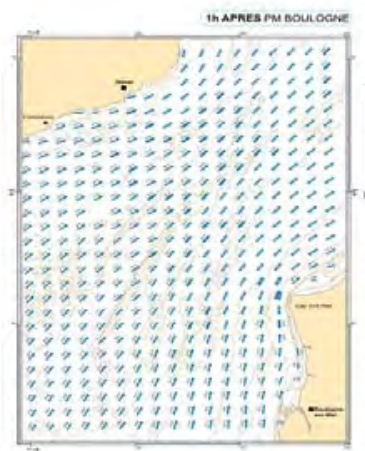

Atlas- édition 2014

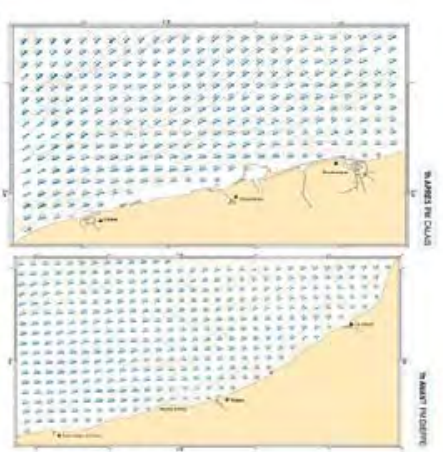

Figure 3. Evolution de la résolution aux abords de ports de Calais, Dunkerque et Boulogne entre les deux éditions de l'atlas 557.

Cet atlas de courant est diffusé sous format imprimé et pdf, téléchargeable sur le site de diffusion du SHOM, ainsi qu'en version numérique destinée aux usages professionnels.

\subsection{Produits de courants de marée 3D}

Le produit courants de marée 3D, résultant de l'exploitation du modèle hydrodynamique (NICOLLE, 2009, 2010a,b \& 2011a,b; DESMARE, 2011 ; DESMARE \& NICOLLE, 2012) représente sur différents niveaux de profondeurs, les vitesses de courants sur un cycle de marée pour une morte-eau et une vive-eau moyenne de coefficients de marée respectifs 45 et 95 .

Ce type de produit fournit pour chaque heure autour d'une pleine mer au port de référence :

- Des fichiers numériques (vectoriels) avec en chaque point d’une grille irrégulière, la hauteur d'eau totale, les vitesses et directions du courant de marée,

- Des cartes géoréférencées représentant les champs de courant (intensité et direction),

- Des cartes de vitesse maximum de courant.

Les formats numériques fournis (ASCII, SHAPE et GeoTIFF) permettent une exploitation facile avec les systèmes d'information géographiques. Sous SIG et autres visualisateurs, ils se représentent sous la forme de points géoréférencés, de vecteurs courants ou d'images.

A ce jour le SHOM compte quatre produits de ce type :

- deux produits commerciaux : Manche, édité en 2013, Iroise-Rade de Brest, édition 2014. Ces produits sont téléchargeables sur demande et bientôt disponibles à la visualisation sur le portail data.shom.fr et téléchargeables sur diffusion.shom.fr. 


\section{Thème 1 - Hydrodynamique côtière}

- deux produits cofinancés par des conventions Etat-Région: Loire (convention Europe-Etat-SHOM et région des pays de la Loire) et Fromveur (convention EtatSHOM et région Bretagne dans le cadre de projet EMR (Energie marines renouvelables). Ces produits devraient être mis à disposition gratuitement via le portail data.shom.fr dans le courant d'année 2014.

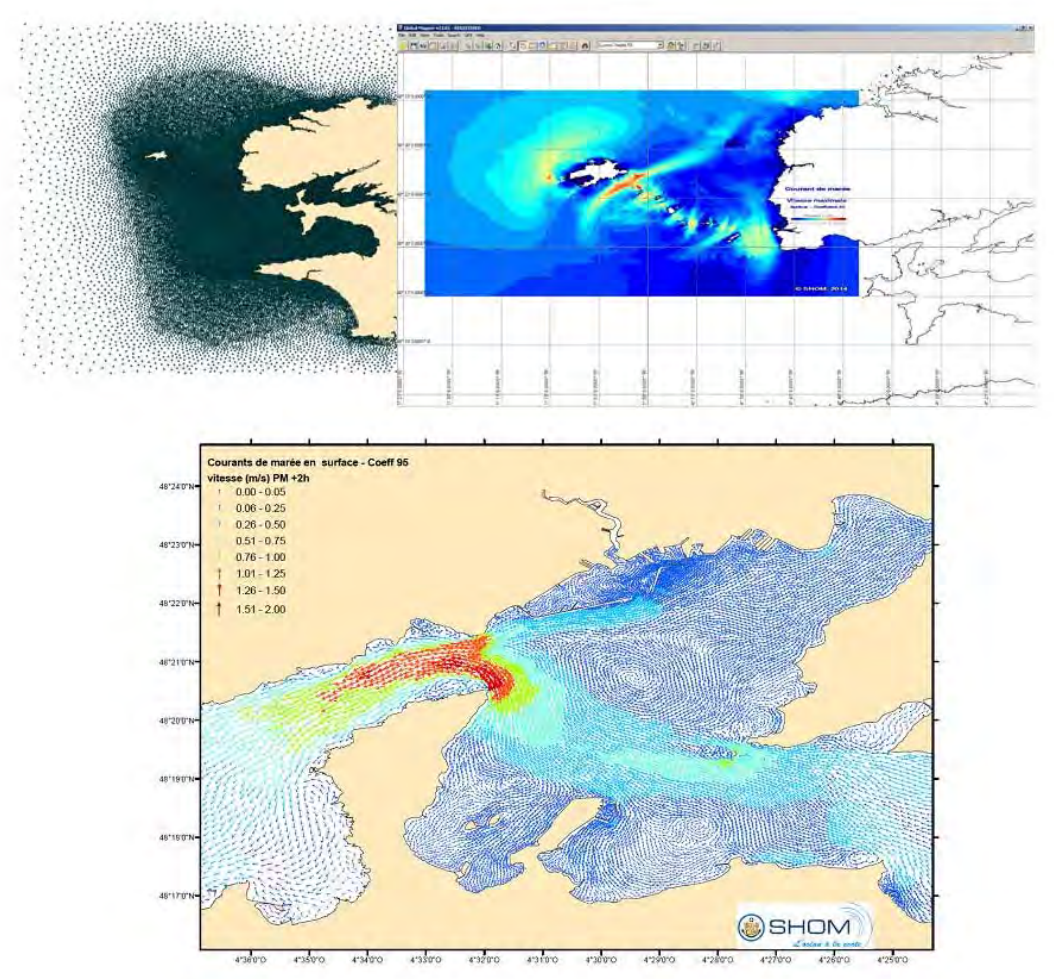

Figure 4. Visualisation d'une carte Geotiff (vitesse maximale en VE en surface pour le produit Fromveur) sous SIG.

Tableau 2. Caractéristiques de différents produits de courant de marée 3D mis en œuvre au SHOM.

\begin{tabular}{|c|c|c|c|}
\hline Produit & Edition & Résolution spatiale & Résolution temporelle \\
\hline Manche & 2013 & surface, mi-profondeur, fond & $\begin{array}{l}1 \text { h de -6h à +6h par rapport à } P M d e \\
\text { Cherbourg }\end{array}$ \\
\hline Fromveur & 2014 & $\begin{array}{l}\text { surface, mi-profondeur, } 10 \mathrm{~m} \\
\text { au-dessus du fond, fond }\end{array}$ & $\begin{array}{l}15 \text { mn de }-6 h 30 \text { à }+6 h 30 \text { par rapport à } \\
\text { PM de Brest }\end{array}$ \\
\hline Loire & 2014 & surface, mi-profondeur, fond & $\begin{array}{l}1 \text { h de -6h à +6h par rapport à } P M d e \\
\text { St Nazaire }\end{array}$ \\
\hline $\begin{array}{l}\text { Iroise-Rade De } \\
\text { Brest }\end{array}$ & 2014 & surface, mi-profondeur, fond & $\begin{array}{l}1 \text { h de -6h à +6h par rapport à } P M d e \\
\text { Brest }\end{array}$ \\
\hline
\end{tabular}




\section{XIII ${ }^{\text {èmes }}$ Journées Nationales Génie Côtier - Génie Civil \\ Dunkerque, 2-4 juillet 2014}

\subsection{Portail de l'information géographique maritime et littorale : data.shom.fr}

Afin de simplifier les modalités d'accès à ses produits et services, le SHOM a mis en place un portail de l'information géographique maritime et littorale de référence, fin janvier 2013. Ce site internet : data.shom.fr permet à tous les usagers (services de l'Etat, collectivités territoriales, entreprises et citoyens) d'accéder aux données de référence, décrivant l'environnement physique maritime, côtier et océanique.

Le portail permet de visualiser les données en ligne, de télécharger des données géographiques maritimes et littorales de référence, et d'y accéder sous forme de flux standardisés (de type WMS, WMTS et WFS).

Les produits du catalogue PPML (Politique Publique Maritime et Littorale) y sont visualisables et téléchargeables (selon les modalités d’accès de ces données).

Un espace de visualisation accessible à tous et des outils d'interaction avec les données permet travailler en ligne et de représenter les couches de données à souhait (voir exemple en figure 5).

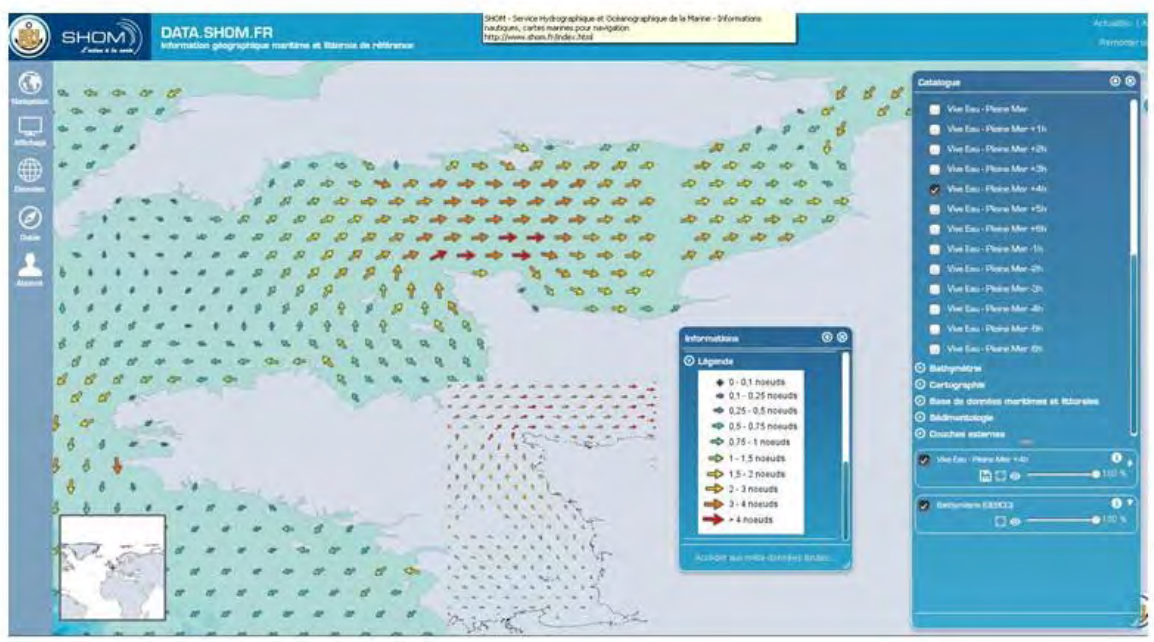

Figure 5. Visualisation des atlas de courant de marée sur le portail data.shom.fr.

\section{Perspectives}

Les perspectives envisagées sont les suivantes :

- En production :

- Les mises à jour des atlas de courant de marée se poursuivent : Baie de Seine en 2014,

- Mise en forme des produits 3D pour fournir les données à meilleure résolution sur les zones couvertes par les modèles,

- Mise à jour des produits 2D et fourniture de formats numériques compatibles avec les outils SIG (shape, geotiff)

- En développement: poursuite de la mise en place de modèles 3D sur la façade Atlantique, selon les ressources disponibles, 
- Concernant le portail data.shom.fr (spécifications en cours) :

- Amélioration des services existants sur le portail de visualisation data.shom.fr,

- Extension des outils proposés avec un service de visualisation des courants de marée avancé, calculs spécifiques destinés aux professionnels,

- Recueil des expressions de besoins et retour d'expérience indispensables sur le nouveau produit 3D pour améliorer et adapter l'offre.

\section{Conclusion}

Cet article avait pour objectif de montrer la gamme de produits de courants de marée actuellement disponibles au SHOM. Ces ouvrages diffusés visent à répondre aux besoins de la navigation, de la plaisance, de la pêche, aux opérations de recherche et de sauvetage et autres activités maritimes. Leurs mises à jours bénéficient des améliorations continues des modèles hydrodynamiques permettant leur conception et d'une meilleure connaissance de l'environnement, avec la densification des réseaux d'observation.

\section{Références bibliographiques}

DESMARE S. (2011). Analyse des besoins et spécifications des produits $3 D$ de Courants de marée. Rapport d'étude SHOM n² 2011-056.

DESMARE S. (2011). Coastal hydrodynamic: Tidal currents modelling application in use at SHOM. Acte de colloque TELEMAC-MASCARET User Conference, Paris.

DESMARE S, NICOLLE A.(2012). Hydrodynamique côtière : modélisation des courants de marée au SHOM (pp 31-40). Acte de colloque JNGCGC 2012, Cherbourg. pp 31-39. http://dx.doi.org/10.5150/jngcgc.2012.004-D

NICOLLE A. (2009). Réalisation d'un modèle 3D de courants de marée dans la rade de Brest. Rapport d'étude SHOM n 2009-176.

NICOLLE A. (2010a). Réalisation d'un modèle 3D de courants de marée dans la Manche. Rapport d'étude SHOM n² 2011-044.

NICOLLE A. (2010b). Réalisation d'un modèle 3D de courants de marée dans la baie de Seine. Rapport d'étude SHOM n² 2011-052.

NICOLLE A. (2011a). Réalisation d'un modèle 3D de courants de marée dans la baie de Somme -Pas de calais. Rapport d'étude SHOM n² 2011-076.

NICOLLE A. (2011b). Réalisation d'un modèle 3D de courants de marée en mer d'Iroise. Rapport d'étude SHOM n 2011-104.

NICOLLE A., PINEAU L. (2009). Modélisation numérique en hydrodynamique côtière. Guide technique SHOM nº 2007-050. 